\title{
Características anatômicas do ser humano nos diferentes períodos da expressão artística
}

\author{
Anatomical characteristics of the human being in different periods of artistic expression \\ Características anatómicas del ser humano en los diferentes períodos de la expresión artística \\ Vitória Imaculada Soares ULIANA ${ }^{1}$ \\ Otavio Marino dos SANTOS NETO \\ João Paulo Mardegan ISSA ${ }^{3}$
}

${ }^{1}$ Graduanda em Farmácia pela Faculdade de Ciências Farmacêuticas de Ribeirão Preto, USP, Universidade de São Paulo, $14040-903$ Ribeirão Preto - SP, Brasil ${ }^{2}$ Doutorando em Odontologia (Reabilitação Oral) pela Faculdade Odontologia de Ribeirão Preto, USP, Universidade de São Paulo, $14040-904$ Ribeirão Preto - SP, Brasil

${ }^{3}$ Professor Associado (Área de Anatomia), Departamento de Biologia Básica e Oral, Faculdade de Odontologia de Ribeirão Preto, USP, Universidade de São Paulo, 14040-904 Ribeirão Preto - SP, Brasil

\section{Resumo}

Esse trabalho foi caracterizado por ser um projeto de cultura e extensão universitária visando expandir os conhecimentos da Universidade de São Paulo para a comunidade. De forma educativa e expositiva, objetivou-se correlacionar aspectos sobre a anatomia e arte, mostrando a importância histórica, desde a época pré-histórica até os dias atuais. O trabalho foi realizado com alunos do Ensino Médio em escolas estaduais do estado de São Paulo, sendo atingidos 210 alunos no total. Os alunos participaram de apresentações que mostravam obras artísticas correlacionadas com peças anatômicas. Observou-se que os resultados condisseram com a realidade da educação brasileira, em que 150 alunos, (74\%) não dedicavam nenhum tempo fora da escola para o estudo da arte. Constatou-se que os alunos tinham informações sobre o tema (114 alunos, 56\%), as quais obtiveram pela internet e nas aulas de artes, demonstrando uma evolução no modo de comunicação e obtenção de informações. Concluiu-se que os alunos se sentiram gratificados pela apresentação, o que foi observado pela análise qualitativa realizada com o instrumento de avaliação.

Descritores: Anatomia; Arte; Ensino Fundamental e Médio.

\section{Abstract}

This work was characterized by being a project of culture and university extension aiming to expand the knowledge of the University of São Paulo to the community. In an educational and expository manner, the objective was to correlate aspects of anatomy and art, showing the historical importance, from prehistoric times to the present day. The work was carried out with high school students in state schools of the state of São Paulo, reaching 210 students in total. Students participated in presentations that showed artwork correlated with anatomical pieces. The results were consistent with the reality of Brazilian education, in which 150 students (74\%) did not devote any time away from school to study art. It was found that the students had information on the subject (114 students, 56\%), which they obtained through the internet and in art classes, demonstrating an evolution in the way of communication and obtaining information. It was concluded that the students felt gratified by the presentation, which was observed by the qualitative analysis performed with the assessment instrument.

Descriptors: Anatomy; Art; Education, Primary and Secondary.

\section{Resumen}

Este trabajo se caracterizó por ser un proyecto de cultura y extensión universitaria con el objetivo de expandir el conocimiento de la Universidad de São Paulo a la comunidad. De manera educativa y expositiva, el objetivo era correlacionar aspectos de la anatomía y el arte, mostrando la importancia histórica, desde la prehistoria hasta la actualidad. El trabajo se realizó con estudiantes de secundaria en escuelas públicas del estado de São Paulo, llegando a 210 estudiantes en total. Los estudiantes participaron en presentaciones que mostraron obras de arte correlacionadas con piezas anatómicas. Los resultados fueron consistentes con la realidad de la educación brasileña, en la cual 150 estudiantes (74\%) no dedicaron tiempo fuera de la escuela a estudiar arte. Se encontró que los estudiantes tenían información sobre el tema (114 estudiantes, 56\%), que obtuvieron a través de Internet y en clases de arte, lo que demuestra una evolución en la forma de comunicación y la obtención de información. Se concluyó que los estudiantes se sintieron satisfechos con la presentación, que se observó mediante el análisis cualitativo realizado con el instrumento de evaluación.

Descriptores: Anatomía; Art; Educación Primaria y Secundaria.

\section{INTRODUÇÃO}

A arte é uma forma de expressão que sempre esteve presente na história da humanidade, por meio dela são transmitido pensamentos, sentimentos e acontecimentos. Ao longo do tempo a arte tem acompanhado as transformações cientificas, e sua relação com a anatomia é observada pela forma em que o corpo humano tem sido representado em todas as civilizações ${ }^{1}$.

Para que essa representação ocorresse de forma realista, foi necessário um estudo aprofundado na estética humana, assim como também na anatomia. Nesse aspecto, artistas do período Renascentista do século XV e XVI como Leonardo da Vinci, Michelangelo e Sandro Botticelli possuíam a preocupação de absorver conhecimentos ligados ao corpo humano, para que assim pudessem transmitilos em suas obras ${ }^{2}$.

O conhecimento das civilizações antigas se deve ao fato de descobertas no ramo artístico. A figura da Vênus de Willendorf, 24 mil a.C, por exemplo, mostra a primeira representação do corpo feminino, evidenciando o corpo da mulher acima do peso o que representava fertilidade para a época. Uma representação anatômica deste corpo nos leva a acreditar que o ser humano não estaria preocupado em representar de forma realista o corpo humano, isso porque não havia estudos anatômicos tão aprofundados como aconteceu na Renascença ${ }^{3}$.

Leonardo da Vinci (1452-1519) no período renascentista rompeu os limites da arte e da ciência, pois além de um grande pintor em seu tempo, interessava-se pelo estudo da anatomia, botânica, física, química e outros estudos científicos. Porém a área de estudo na qual ele mais se dedicou foi a anatomia humana, como foi demonstrado na exposição Leonardo da Vinci "Anatomist", que reuniu mais de 200 desenhos feitos pelo pintor do corpo humano, e no estudo "A natureza, a razão e a ciência do homem: edição dos estudos de anatomia de Leonardo da Vinci", do historiador Eduardo Kickhöfel professor da Universidade Federal de São Paulo (Unifesp) . Os estudos feitos por Da Vinci 
resultaram em descobertas importantes sobre arteriosclerose, por exemplo, promovendo uma evolução na medicina e trazendo para a área artística um caráter cientifico ${ }^{4}$.

Nesse contexto, objetivo deste trabalho foi abordar as diferentes expressões e características anatômicas dos seres humanos, através de um modelo teórico expositivo que correlacionou as estruturas anatômicas, expressões e posturas corporais com a percepção dos artistas ao longo do tempo. Através da difusão cultural, buscou-se uma maior aproximação dos estudantes do ensino médio com o ambiente universitário, causando uma sinergia entre ciências da saúde e ciências humanas.

\section{MATERIAL E MÉTODO}

As atividades foram realizadas fora do ambiente universitário, para alunos do ensino médio da rede pública do estado de São Paulo, e englobou duas cidades: Ribeirão Preto e Mirassol. Inicialmente foram realizados contatos com as escolas, através de seus coordenadores pedagógicos, que permitiram o desenvolvimento das atividades. Uma apresentação de slides foi realizada utilizando o programa Microsoft Power Point, apresentando imagens das obras de arte, peças anatômicas e questões de vestibulares vinculadas ao assunto. Foi utilizada a linguagem cientifica juntamente com a linguagem artística justamente para concretizar a interdisciplinaridade entre a ciência e a arte. Preconizou-se que apresentação se desse de forma didática e dinâmica, mostrando a evolução no âmbito artístico e cientifico ao longo do tempo, para a melhor compreensão dos alunos.

A obtenção dos dados foi realizada pela aplicação de questionário com perguntas acerca da apresentação e do tema, que permitiu obter dados do público atingido. (Quadro 1).

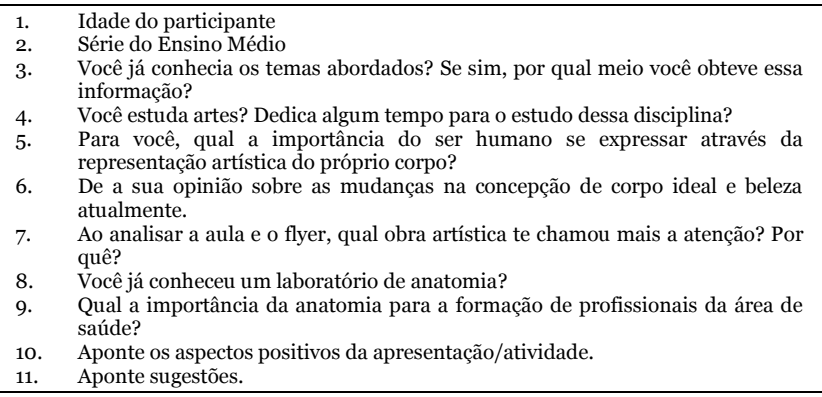

Quadro 1: Questionário com perguntas a respeito do tema abordado pelo projeto.

\section{RESULTADOS}

O trabalho foi realizado em três escolas, dentre elas Escola Estadual Alpheu Dominiguetti em Ribeirão Preto, Escola Estadual Dom Alberto José Gonçalves em Ribeirão Preto e Escola Genaro Domarco em Mirassol, todas localizadas no estado de São Paulo. Foram englobados 210 alunos, sendo que destes 202 responderam aos questionários. A partir dos questionários obtidos, a maioria dos alunos que participaram do trabalho cursavam o $3^{\circ}$ ano do Ensino Médio (cerca de 56\%) e tinham em média 17 $\operatorname{anos}(37 \%)$ e 16 anos (35\%).

Analisou-se que a maioria dos alunos já tinham visto parte das obras em algum lugar na internet ou até mesmo na escola em aulas de artes (cerca de 114 alunos 56\%), denotando conhecimento prévio sobre o assunto (cerca de 74 alunos 37\%). Entretanto, muitos não tinham conhecimento do significado da palavra "anatomia" e como ela poderia estar relacionada com a arte (Figura 1). Também foi analisado através do questionário que os alunos não estudavam artes fora da escola ou não dedicavam tempo no estudo dessa disciplina (150 alunos, 74\%) conforme demonstrado no gráfico da Figura 2. Além disso, poucos alunos tiveram a oportunidade de visitar um laboratório de anatomia, 11 alunos (5\%) já visitaram e 191 alunos (95\%) ainda não haviam visitado (Figura 3).

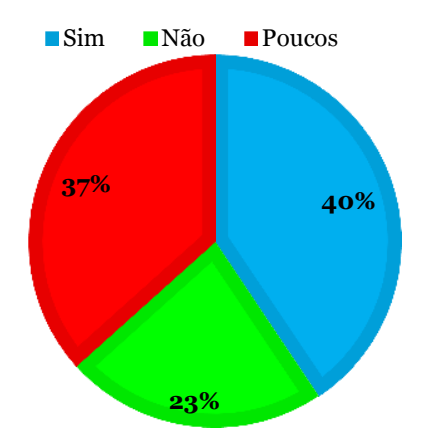

Figura 1: Distribuição da frequência de alunos que já tinham tido contado com o tema proposto.

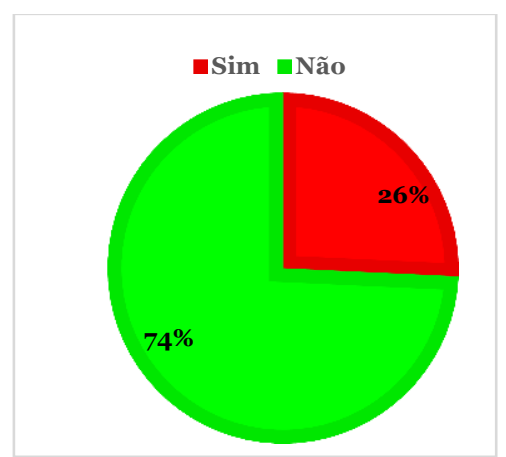

Figura 2: Distribuição da frequência de alunos que estudam arte fora do ambiente escolar.

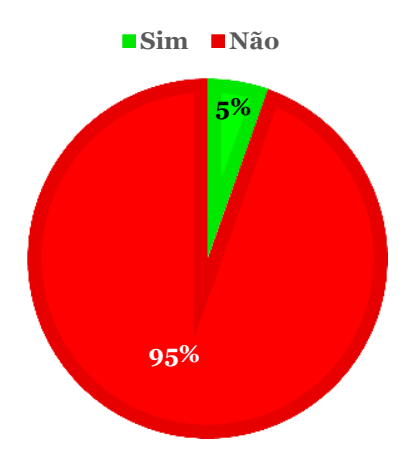

Figura 3: Distribuição da frequência de alunos que já visitaram um laboratório de anatomia.

Durante a apresentação foi observado que 
muitos alunos se mantinham atentos à aula e tentavam compreender ao máximo o que estava sendo exposto. A maioria deles conseguiu entender e responder os exercícios de vestibular que foram levados para melhor fixação de conhecimento. Pelas críticas obtidas pelos 202 questionários, foi observado que grande parte dos alunos avaliou a aula como esclarecedora e didática. Foi possível a conscientização sobre a importância do estudo de anatomia nos diferentes períodos de nossa sociedade e sua indispensabilidade na formação de profissionais da saúde, que necessitam de estudos anatômicos para a formação profissional.

Observou-se que os alunos tiveram uma simpatia maior com as obras mais famosas que são geralmente trabalhadas em sala de aula, como por exemplo "Mulher Chorando" de Pablo Picasso e "O Homem Vitruviano" de Leonardo Da Vinci. Muitos alunos ficaram impressionados com as análises anatômicas feitas em sala de aula e como o tema está relacionado com o cotidiano.

Em um aspecto critico, foi trabalhado a percepção dos alunos perante as mudanças ocorridas com os padrões de beleza ao longo dos anos, como a figura da vênus de Willendorf, antes considerada uma deusa da fertilidade e na atualidade julgada por estar fora dos padrões estéticos de beleza, o que denotou a fluidez do padrão de beleza que pode mudar constantemente ao longo dos anos.

\section{DISCUSSÃO}

Nos aspectos pedagógicos o trabalho realizado tentou trazer atividades que valorizassem o erro e os acertos para os alunos, para que assim a aprendizagem ocorresse através da ação experimental. "O indivíduo constrói através do fazer e refazer das atividades, estando a educação a serviço da causa social. A aprendizagem é feita através da ação experimental e da valorização do erro e do acerto" essa é a maneira de como as crianças e adolescentes consumam seu conhecimento. Dessa forma, este trabalho fez a tentativa de trazer aos alunos o ânimo para responder as questões de maneira correta, dando dicas para que estes pudessem buscar em suas memórias a resposta exata, além de valorizar o erro e dar incentivo ao aluno para tentar novamente ${ }^{5}$.

Os estudos anatômicos são de extrema importância para os jovens que possuem interesse em seguir profissões ligadas à área da saúde, sendo considerados essenciais para que o estudante consiga se preparar, identificar e reconhecer as funções orgânicas em seus aspectos corporais e patológicos. Esses estudos podem ser feitos de três maneiras: com atlas, textos figuras e cadáveres ${ }^{6}$.

Já os estudos artísticos são importantes por fornecer uma nova perspectiva e despertar uma forma de observar o mundo com outros olhos, além de demonstrar a grande interligação com outras áreas e promover a criatividade. Pôde-se observar que ainda prevalece nas escolas o ensino de artes com técnicas de desenho geométrico e de observação, considerado atualmente um método antiquado de aprendizagem ${ }^{7}$. No trabalho foi observado uma defasagem dos alunos em artes. Aqueles que estavam mais familiarizados possuíam também maior facilidade em observar e tirar conclusões daquilo que lhe foi proposto, mostrando que havia uma melhor percepção do que estava acontecendo, o que infelizmente não foi frequente. As obras das quais os alunos mais se identificaram são também as mais difundidas pelas aulas de artes, mídia ou internet como o Homem Vitruviano" de Leonardo da Vinci, "Mulher Chorando" de Pablo Picasso, e também a "Criação de Adão" de Michelangelo. Logo, para a melhor compreensão da interdisciplinaridade entre arte e anatomia, foi feita uma linha do tempo que englobava diversas culturas e demonstrava aos alunos como a evolução cientifica está intrinsicamente ligado ao tema. O trabalho utilizou-se de obras de diversos artistas que tinham obras ligadas à anatomia, com o objetivo de buscar uma correlação entre arte e anatomia. $\mathrm{O}$ ensino de anatomia humana, por exemplo, para os cursos de ciências médicas necessita de métodos pedagógicos mais satisfatórios e atraentes, devido a real necessidade desse conhecimento na atividade profissional ${ }^{8}$.

A representação artística explora nas diferentes áreas gráficas as vias mais instintivas de processamento do cérebro humano, dado às formas e dimensões, mais do que descrições verbais ou textuais. A conjunção das duas formas de expressão confere um enorme poder de comunicação aos temas tratados ${ }^{9}$.

O trabalho realizado demonstra que a anatomia e arte estão em constante interdisciplinaridade. Grandes autores contemporâneos como Guther Von Hagers fazem dissecação em corpos e os coloca como se fossem vivos, demonstrando a união com o estudo anatômico e a representação artística da qual esse tipo de trabalho é feito ${ }^{10}$. Presumimos que a união da anatomia, arte e até mesmo a ciência, permite um melhor entendimento de estruturas e funções do corpo, expandindo conceitos científicos.

\section{CONCLUSÃO}

Pela metodologia empregada foi possível fazer uma avaliação qualitativa positiva entre a associação do ensino de artes com a anatomia. Dessa forma, o entendimento artístico de questões ligadas ao corpo humano, além de promover o questionamento técnico-científico, foi capaz de promover uma importante correlação com os padrões socioculturais ao longo do tempo, promovendo a difusão de conhecimentos através da extensão universitária. 


\section{CONFLITO DE INTERESSES}

Os autores declaram não haver conflitos de interesse.

Os autores agradecem a Pró-Reitoria de Cultura e Extensão Universitária (PRCEU-USP) e ao Banco Santander pelo financiamento das atividades de extensão relatadas nesse trabalho - $1^{\circ}$ Edital Santander / USP / FUSP de Fomento às Iniciativas de Cultura e Extensão.

\section{REFERÊNCIAS}

1. Azevedo Junior J. Apostila de Arte - Artes Visuais. São Luís: Imagética Comunicação e Design; 2007.

2. Isaacsson W. Livro Leonardo da Vinci. São Paulo: Intrínseca; 2017.

3. Baumgart TF. Breve História da Arte. São Paulo: Martins Fontes; 1999.

4. Haag C. Entre a cátedra e o ateliê. Agosto 2012. Disponível em http://revistapesquisa.fapesp.br/2012/08/10/entrea-c\%C3\%A1tedra-e-o-ateli\%C3\%AA/>. Acesso em 12 de fevereiro de 2019.

5. Nascimento MEPA. Pedagogia Freinet: Natureza, Educação e Sociedade. Campinas, SP: Editora da Unicamp; 1995.

6. Infantosi AFC, Klemt A. Visualização 3D da dissecção crânio: a surfasse method for visualising the $3 \mathrm{~d}$ dissection of the human skull. Rev Bras Eng Bioméd. 2000;16(1):27-37.

7. Barbosa AM. Teoria e prática da educação artística. São Paulo: Cultrix; 1975

8. Fraher JP; Evans DJ. Training tomorrow's anatomists today:a partnership approach. Anat Sci Educ. 2009;2(3):119-25.

9. Adro CO. Anatomia comparada da articulação temporomandibular [dissertação]. Lisboa: Faculdade de Belas Artes, Universidade de Lisboa; 2011.

10.Rebollo RA. "De humani corporis circus" de Gunther von Hagens. Scientiæ zudia. 2003;1(1):101-7.

\section{AUTOR PARA CORRESPONDENCIA}

\section{João Paulo Mardegan Issa}

jpmissa@forp.usp.br

\section{Submetido em 26/07/2019
Aceito em 29/11/2019 \\ Submetido em 26/07/2019
Aceito em 29/11/2019}

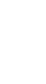

